\title{
Flexible Erwerbsverläufe und Sozialversicherung
}

Erwerbsunterbrechungen, aber auch Phasen atypischer Beschäftigung, haben in den letzten Jahrzehnten in Europa an Bedeutung gewonnen. Die Renten- und Arbeitslosenversicherungssysteme europäischer Länder sind bislang unterschiedlich gut auf diese Entwicklungen vorbereitet. Dies zeigt insbesondere der Vergleich zwischen den sozialversicherungsbasierten kontinentaleuropäischen Ländern mit hoher Äquivalenz zwischen Beiträgen und Leistungen und den nordischen Ländern sowie den Niederlanden mit stärker individualisierten Leistungen und Grundsicherungssystemen. Dieser Beitrag diskutiert Anpassungsstrategien für die Sozialversicherungsländer.

\section{1 \\ Einleitung}

Die zunehmende Erwerbstätigkeit von Frauen, die Fragen der Vereinbarkeit von Familie und Beruf aufwirft, sowie der Anstieg atypischer Erwerbsformen haben in den letzten Jahrzehnten zu einer stärkeren Flexibilisierung von Erwerbsverläufen geführt. Diese Entwicklung stellt die sozialen Sicherungssysteme vor neue Herausforderungen.

Insbesondere in der Gruppe der kontinentaleuropäischen Länder, ${ }^{1}$ die wesentlich auf dem Sozialversicherungsmodell beruhen, sind die sozialen Sicherungssysteme traditionell stark auf das „männliche Ernährermodell“ ausgerichtet. Diesem liegt das sogenannte Normalarbeitsverhältnis von Männern zugrunde, das durch abhängige, dauerhafte Vollzeiterwerbstätigkeit bei einem Arbeitgeber charakterisiert ist. (Ehe)Frauen wird in diesem Modell die Rolle der Vollzeitbetreuerin und Hausfrau mit allenfalls geringfügigem Zuverdienst zugeschrieben. Soziale Sicherungsleistungen stellen auf Statussicherung ab und weisen eine starke Äquivalenz zwischen Beiträgen und Leistungen auf. Darüber hinaus werden abgeleitete Leistungen wie die kostenlose Familienmitversicherung in der gesetzlichen Krankenversicherung (GKV) und die Hinterbliebenenrenten für Witwen und Waisen in der gesetzlichen Rentenversicherung (GRV) gewährt.

Das männliche Ernährermodell entspricht heute in den kontinentaleuropäischen Ländern weder den Präferenzen der Bevölkerung ${ }^{2}$ noch ist ein System mit abgeleiteten Leistungen angesichts immer weniger (stabiler) Ehen und hoher Anteile von Alleinerziehenden adäquat und nachhaltig existenzsichernd. Außerdem ist eine starke Äquivalenz zwischen Beiträgen und Leistungen angesichts der ungleichen Verteilung von Betreuungs- und Haushaltsaufgaben bei gleichzeitig fehlenden kompensierenden Institutionen (insbesondere umfassende Klein- und Schulkinderbetreuung) nicht verteilungsgerecht

Dieser Beitrag diskutiert, inwiefern die Renten- und Arbeitslosenversicherungssysteme anderer europäischer Länder flexiblen Erwerbsverläufen besser gerecht werden und Reformoptionen für Deutschland und andere kontinentaleuropäische Länder bieten.

In Abschnitt 2 wird auf die zunehmende Flexibilisierung von Erwerbsverläufen eingegangen. Der Fokus liegt auf Erwerbsunterbrechungen aufgrund der Nichtvereinbarkeit von Familie und Beruf und auf atypischen Erwerbsformen - insbesondere Teilzeitbeschäftigung und befristeter Beschäftigung. Die Abschnitte 3 und 4 diskutieren die Probleme von Personen mit flexiblen Erwerbsverläufen beim Zugang zu Leistungen und bei der Leistungshöhe. Erörtert werden auch gute Praktiken aus einer Reihe europäischer Länder. Schlussfolgerungen zieht Abschnitt 5.

\section{2 \\ Flexible Erwerbsverläufe}

\subsection{VEREINBARKEIT VON FAMILIE UND BERUF}

Die Ausprägungen flexibler Erwerbsverläufe, die auf Erwerbsunterbrechungen oder Verkürzung der Arbeitszeit beruhen und im Zusammenhang mit Betreuungsaufgaben stehen, variieren stark zwischen europäischen Ländern (OECD 2009a). Die Unterschiede sind insbesondere auf die Ausgestaltung der vereinbarkeitsfördern- den Institutionen (insbesondere Kinderund Altenbetreuung, aber auch Elternzeitund Teilzeitregelungen sowie flexible Arbeitszeitarrangements) sowie die Beteiligung von Männern an Betreuungsaufgaben zurückzuführen. Diese Faktoren werden in starkem Maße von den vorherrschenden Werten und Normen geprägt (Leschke/Jepsen 2009).

Dabei sind die kontinentaleuropäischen Länder (und in noch stärkerem Maße die südeuropäischen Länder) im Gegensatz zu den nordischen (und in abgeschwächter Form den angelsächsischen) Ländern durch vergleichsweise konservative Werte und Normen gekennzeichnet. ${ }^{3}$ Betreuungs- und Haushaltsaufgaben wurden lange Zeit als exklusive Aufgabe von Frauen betrachtet und werden noch immer

\footnotetext{
Belgien, Deutschland, Frankreich, Luxemburg und Österreich werden üblicherweise als kontinentaleuropäische Länder eingeordnet, teilweise auch die Niederlande, die aber in einer Reihe von Merkmalen zunehmend den nordischen Ländern ähnlicher werden als den kontinentaleuropäischen.

2 Im Jahr 2001 bevorzugten nur zwischen 3,9\% (Österreich) und 14,1 \% (Frankreich) der Paare mit Kindern unter sechs Jahren ein Modell, bei dem der Mann Vollzeit arbeitet und die Frau nicht beschäftigt ist. Gleichzeitig entsprach diese Arbeitsteilung in 27,3 \% (Belgien) bis 52,3\% (Deutschland) der Paarhaushalte mit Kindern unter sechs Jahren der Realität (OECD 2001, S. 135).

3 Während in Schweden und Dänemark ca. 40 \% der männlichen Arbeitnehmer jeden Tag eine Stunde oder mehr Haushaltsaufgaben verrichten, belaufen sich die Anteile für Deutschland und Österreich auf $18 \%$ und $13 \%$. Bei den Arbeitnehmerinnen gibt es mit knapp über $70 \%$ nur sehr geringe Unterschiede zwischen den vier Ländern (European Foundation 2005, eigene Auswertung).
}

Janine Leschke, Dr., ist Wissenschaftlerin am Europäischen Gewerkschaftsinstitut (EGI) in Brüssel. Arbeitsschwerpunkte: Arbeitsmarkt- und Sozialpolitik.

e-mail: jleschke@etui.org 
durch steuerliche Begünstigungen der Nicht- oder geringfügigen Erwerbsbeteiligung (z. B. in Form von Minijobs), durch Möglichkeiten für vergleichsweise lange Erwerbsunterbrechungen und Förderung von Teilzeit unterstützt. Externe Betreuungseinrichtungen, die Frauen insbesondere in nordischen Ländern eine freie Wahl zwischen Erwerbstätigkeit und Nichterwerbstätigkeit sowie Teilzeit- und Vollzeitbeschäftigung ermöglichen, nahmen in diesem Modell eine subsidiäre Position ein. Dies spiegelt sich auch heute noch in Deutschland und Österreich in geringen Betreuungsquoten bei Kleinkindern und in Halbtagsschulsystemen ohne umfassende Nachmittagsbetreuung wider. Insbesondere aufgrund von langen und teilweise unumkehrbaren Erwerbsunterbrechungen sind die Beschäftigungsquoten von Frauen in kontinentaleuropäischen Ländern trotz der starken Zuwächse weiterhin deutlich geringer als in den nordischen Ländern und etwa den Niederlanden. ${ }^{4}$

Aufgrund der sinkenden Geburtenraten, die teilweise auf die Nichtvereinbarkeit von Familie und Beruf zurückgeführt werden können, ${ }^{5}$ und der daraus resultierenden gesellschaftlichen Kosten (einschließlich der Finanzierungsprobleme sozialer Sicherungssysteme) fand in Deutschland in der letzten Legislaturperiode ein Umdenken statt. Es manifestiert sich in einem verstärkten Ausbau der Klein- und Schulkinderbetreuung sowie in einem neuen Elternzeitrecht, das die kontinuierliche Erwerbsbeteiligung von Müttern fördert und Männer stärker an Betreuungsaufgaben beteiligt.

\subsection{ATYPISCHE BESCHÄFTIGUNG}

Vor dem Hintergrund steigender Erwerbsquoten insbesondere von Frauen haben atypische Beschäftigungsformen in den letzten 15 Jahren an Bedeutung gewonnen. So stieg die Teilzeitbeschäftigung im EU-15Durchschnitt zwischen 1992 und 2007 von $14,2 \%$ auf 20,9\%. Die Frauenteilzeitquote stieg um ca. 8 Prozentpunkte auf heute $36,7 \%$ (Eurostat 2008). Ein - wenn auch weniger starker - Anstieg ist auch bei befristeten Beschäftigungsverhältnissen zu verzeichnen. Die Quote erhöhte sich im gleichen Zeitraum von 11,2 \% auf 14,8\%, die Frauenquote liegt um etwa 1,5 Prozentpunkte höher als die Männerquote (Eurostat 2008). Sowohl Teilzeitarbeit als auch befristete Beschäftigungsverhältnisse en- den häufiger in Arbeitslosigkeit oder Inaktivität als Vollzeitarbeit und unbefristete Beschäftigung. Sie können somit zusätzlich zu den Erwerbsunterbrechungen aufgrund von Betreuung zu instabilen und flexiblen Erwerbsverläufen beitragen (European Commission 2003, S. 131f.; Leschke 2008, S. 140f.; Schulze Buschoff/Protsch 2008, S. 56ff.). In welchem Maße atypische Beschäftigungsverhältnisse Übergänge in reguläre Jobs ermöglichen oder aber dauerhaft suboptimale Beschäftigungsoptionen mit höherem Risiko von Arbeitslosigkeit und Inaktivität darstellen, variiert nicht nur zwischen einzelnen Ländern, sondern auch zwischen verschiedenen Arbeitsmarktgruppen (O’Reilly/Bothfeld 2002).

Atypische Erwerbsformen wurden sowohl auf europäischer Ebene im Rahmen der Europäischen Beschäftigungsstrategie als auch in einzelnen Ländern aktiv durch Steueranreize, (De-)Regulierungsmaßnahmen und verschärfte Arbeitswiederaufnahmekriterien befördert. In Deutschland beispielsweise führten Maßnahmen wie die Neuregelung der Minijobs und die Einführung der Ich-AGs (heute: Gründungszuschuss) zu mehr geringfügiger Beschäftigung und mehr Solo-Selbstständigkeit mit fehlendem oder eingeschränktem Versicherungsschutz bei gleichzeitigem Rückgang der sozialversicherungspflichtigen Beschäftigung (Bundesagentur für Arbeit 2006; 2007). Auch wird Teilzeitbeschäftigung weiterhin als adäquate Lösung für die Vereinbarkeit von Familie und Beruf dargestellt und in dieser Funktion - häufig aufgrund fehlender Kinderbetreuungseinrichtungen - fast ausschließlich von Frauen ausgeübt.

Bei Frauen kumulieren verschiedene Risiken, die zu flexiblen Erwerbsverläufen führen können. Diese flexiblen Erwerbsverläufe haben aufgrund der Ausrichtung der Sicherungssysteme auf das sogenannte Normalarbeitsverhältnis insbesondere in kontinentaleuropäischen Ländern negative Folgen für die soziale Absicherung der betroffenen Gruppen. ${ }^{6}$

\section{Alterssicherungssysteme}

\subsection{TRENDS IN DEN RENTEN- SYSTEMEN}

Die Mehrheit der europäischen Länder hat in den letzten Jahren ihre Alterssicherungs- systeme reformiert. Dabei stand meist die finanzielle Nachhaltigkeit im Vordergrund. Unabhängig von der Reichweite der einzelnen Reformen lassen sich gemeinsame Trends erkennen: In der GRV (erste Säule) fand vielfach eine stärkere Verknüpfung zwischen Beiträgen und Leistungen statt, während gleichzeitig die betriebliche Altersvorsorge (zweite Säule) und die individuelle Zusatzversicherung (dritte Säule) gestärkt wurden. Dies erschwert eine Umverteilung innerhalb des Systems. Ein weiterer Trend ist die Abschaffung oder starke Einschränkung der Hinterbliebenenrenten zugunsten stärker individualisierter Systeme. Abgeleitete Hinterbliebenenleistungen sind nicht nur kostenintensiv, sondern sie setzen auch Negativanreize für eine Arbeitsmarktbeteiligung. In Deutschland spielen sie trotz der Reformen des Jahres 2001 noch immer eine wichtige Rolle (Klammer 2005, S. 352f.).

Die Reformen der letzten Jahre führten in fast allen Ländern, Deutschland eingeschlossen, zu Leistungseinbußen für Geringverdiener. ${ }^{7}$ Eine Ausnahme bildet das Vereinigte Königreich. Verbesserungen für Geringverdiener sind auf die Einführung einer zusätzlichen Staatsrente (state second pension) je nach Einkommen variierenden Lohnersatzraten sowie auf eine Erhöhung der Mindesteinkommensgarantie, jetzt pension credit, zurückzuführen (OECD 2007b, S. 66f.). Laut Berechnungen des EU-Ausschusses für Sozialschutz (Social

4 Die Beschäftigungsquoten von Frauen liegen in den kontinentaleuropäischen Ländern zwischen $55,7 \%$ in Belgien und 65,7 \% in Österreich. In den nordischen Ländern liegen sie zwischen $70 \%$ in Finnland und $74,4 \%$ in Dänemark (Eurostat 2008).

5 In Europa existiert heute ein positiver Zusammenhang zwischen Beschäftigungsquoten und Geburtenraten (OECD 2007a, S. 35f.).

6 So zeigen die Daten der Studie "Altersvorsorge in Deutschland" (AVID) 2005 einen klaren Zusammenhang zwischen Erwerbsbiografien und Alterseinkommen. Je mehr Jahre eine Person Vollzeit gearbeitet hat, umso höher sind die projizierten Rentenanwartschaften. Eindeutig negative Zusammenhänge ergeben sich hingegen zwischen geringfügiger Beschäftigung und Alterseinkommen, Zeiten der Arbeitslosigkeit und Alterseinkommen sowie längeren Kindererziehungszeiten und Alterseinkommen (Riedmüller/Willert 2008, S. 11f.)

7 In Deutschland lagen die Rentenansprüche eines Arbeitnehmers mit vollständiger Karriere und der Hälfte des durchschnittlichen Einkommens vor den Reformen leicht unterhalb des OECD-Durchschnitts von $40 \%$ des durchschnittlichen Nettoeinkommens und nach den Reformen bei $32 \%$ (OECD 2007b, S. 128f.). 
Protection Committee 2006, S. 22f.) werden die Reformen (in ca. einem Viertel der EU-27-Länder) die Nettolohnersatzraten von Personen mit Erwerbsunterbrechungen (definiert als Erwerbsbiografie mit 30 von 40 Erwerbsjahren) stärker senken als jene von Personen ohne Erwerbsunterbrechungen. Im Großteil der EU-27-Länder sind die Reformfolgen für beide Gruppen vergleichbar - allerdings basierend auf einem niedrigeren Ausgangsniveau für Personen mit Erwerbsunterbrechungen.

\subsection{DIE ERSTE STAATLICHE SÄULE}

In der ersten Säule bewirken einerseits Mindestbeitragszeiten und andererseits die Koppelung des Rentenniveaus an Beitragszeiten und die Höhe des Arbeitseinkommens Unterschiede im Leistungsniveau zwischen flexibel Beschäftigten und „Normalarbeitnehmern".

Die deutsche Rentenformel in der GRV orientiert sich beispielsweise stark an der Dauer der vorherigen Erwerbstätigkeit und an der Höhe des versicherungspflichtigen Erwerbseinkommens, was dazu führt, dass die durchschnittlichen eigenständigen Renten von Frauen in Westdeutschland 2004 nicht einmal halb so hoch wie die von Männern waren. In Ostdeutschland lagen sie bei zwei Dritteln. Trotz des Anstiegs der Erwerbsbeteiligung von Frauen waren in dieser Hinsicht in Westdeutschland seit den 1960er Jahren aufgrund der starken Verbreitung der Teilzeitbeschäftigung von Frauen keine Verbesserungen zu beobachten (Klammer 2005, S. 349).

\subsection{MINDESTBEITRAGSZEITEN UND KOPPELUNG VON BEITRAGSZEITEN UND RENTENHÖHE}

Bereits die Mindestbeitragszeit, die den $\mathrm{Zu}$ gang zu Rentenversicherungssystemen bestimmt, kann in einer Reihe von EU-Ländern ein Problem für Personen mit Erwerbsunterbrechungen darstellen. So liegen die Mindestbeitragszeiten in neun Ländern bei mindestens 15 Jahren, in drei weiteren bei zehn Jahren. Bei der zweiten Gruppe handelt es sich ausschließlich um mittel- und osteuropäische Mitgliedsländer sowie südeuropäische Länder (MISSOC 2008a). ${ }^{8}$ Außerdem werden volle Renten häufig erst bei vergleichsweise langen Beitragszeiten gezahlt. Dies gilt auch für Länder mit relativ kurzen Mindestbeitragszeiten wie Deutschland. In den meisten
Ländern werden bestimmte Arten der Erwerbsunterbrechung für die Rentenberechnung als Beitragszeiten gewertet. Beispiele sind kurzfristige Arbeitslosigkeit, Elternzeit, aber in einigen Fällen auch Kindererziehung, die über die Elternzeit hinausgeht, Krankheit oder Weiterbildung. ${ }^{9}$ Problematisch für die Rentenhöhen ist allerdings, dass diese Zeiten häufig nur auf der Basis von Mindestbeiträgen einbezogen werden (Europäische Kommission 2004, S. 91ff.).

Wichtig ist in diesem Zusammenhang, dass Gutschriften (insbesondere für die Betreuung von Kindern) geschlechtsneutral gewährt werden und keine Negativanreize zur Arbeitsbeteiligung setzen, da sie sonst die geschlechtertypische Arbeitsteilung weiter befördern und sich negativ auf die Karrieren von Frauen auswirken können. Das deutsche und schwedische Beispiel können hier positiv hervorgehoben werden, da die Gutschrift nicht an eine Unterbrechung der Erwerbstätigkeit geknüpft ist. In Deutschland werden die Entgeltpunkte für die Kindererziehung und die Entgeltpunkte aus einer Beschäftigung bis zur Beitragsbemessungsgrenze addiert (BMFSFJ 2002, S. 26, 27).10 In Schweden kann für maximal die ersten vier Lebensjahre eines Kindes zwischen drei Optionen bei der Aufstockung von Beiträgen gewählt werden: Beiträge basierend auf $75 \%$ der Durchschnittseinkommen aller Versicherten, Beiträge basierend auf $80 \%$ des individuellen Einkommens im Jahr vor der Geburt des Kindes oder Inanspruchnahme eines festgelegten Zuschlags (Ståhlberg et al. 2005, S. 66; Palmer 2001, S. 7).

\subsection{KOPPELUNG VON ARBEITS- EINKOMMEN UND RENTENHÖHE}

Um angemessene Renten für flexibel Beschäftigte mit potenziell geringeren oder unstetigen Erwerbseinkommen zu garantieren, bieten sich Grund- oder Mindestrentensysteme an. Sie existieren nur in einigen europäischen Ländern. Zudem unterscheiden sich die Leistungen stark in der Höhe, weshalb sie in manchen Ländern nicht armutvermeidend wirken. Üblicherweise werden sie von beitragsbasierten Zusatzsystemen begleitet (MISSOC 2008b). In den nordischen Ländern und in den Niederlanden existieren universelle steuerfinanzierte Systeme, die auf dem Wohnsitzprinzip beruhen und keine Nachteile für flexibel Beschäftigte beinhalten. Die Grundrente im Vereinigten Königreich be- schränkt sich auf abhängig Erwerbstätige und Selbstständige und wird nur ausgezahlt, wenn eine Mindestanzahl von Beitragsjahren geleistet wurde. Der volle Betrag, der nicht armutvermeidend ist, wird nur bei einer sehr hohen Beitragszeit ausgezahlt, wobei allerdings Kinderbetreuung, Pflege und Arbeitslosigkeit angerechnet werden (MISSOC 2008b). Die Mindestrente in Belgien benachteiligt flexibel Beschäftigte, da sie nur gewährt wird, wenn ein Individuum mindestens zwei Drittel der Karriere vollzeitbeschäftigt war. Außerdem hängt die Leistungshöhe von der Anzahl der gearbeiteten Jahre ab (Peeters et al. 2007, S. 134f.). ${ }^{11}$ Das belgische und das britische Beispiel zeigen, dass die Wirksamkeit von Grundsicherungselementen stark von ihrer Ausgestaltung abhängt.

Die Rentensysteme unterscheiden sich sowohl in der Höhe der Lohnersatzraten als auch im Ausmaß der (linearen) Koppelung an das vorangegangene Erwerbseinkommen. Dementsprechend ist auch der Bedarf für etwaige Zusatzregelungen zur Aufwertung von Renten von Geringverdienern/flexibel Beschäftigten unterschiedlich gelagert (European Commission 2006a, S. 142). Im Folgenden werden einige dieser Zusatzregelungen beschrieben. In Deutschland werden beispielsweise die Rentenanwartschaften von Teilzeitbeschäftigten mit unterdurchschnittlichem Verdienst und Kindern bis zum zehnten Lebensjahr auf maximal $100 \%$ des Durchschnittseinkommens aufgewertet (BMFSFJ 2002, S. 28f.). Auch in Spanien und Griechenland wird Teilzeitbeschäftigung bei der Rentenberechnung anteilig höher bewertet (European Commission 2006a, S. 176 u. 180f.). In Belgien gibt es ebenfalls eine Aufwertungsmöglichkeit, die allerdings nur in sehr speziellen Fällen (z. B. Teilzeit zur Arbeitsumverteilung) zum Tragen kommt und so

\footnotetext{
8 Im Vereinigten Königreich gilt eine zehn- bis elfjährige Beitragszeit für die Grundrente (MISSOC 2008a).

9 Länderbeispiele für die Regelungen zu den einzelnen Erwerbsunterbrechungen werden in MISSOC (2008a, S. 20f.) aufgeführt.

10 Eine längere Nichtbeschäftigung (mehr als drei Jahre) aufgrund von Kindererziehung wirkt sich weiterhin sehr negativ auf die GRV-Anwartschaften aus (Riedmüller/Willert 2008, S. 20).

$1142 \%$ der weiblichen vormaligen Arbeitnehmer hatten keinen Anspruch auf die garantierte Mindestpension, während dies nur bei $3 \%$ der männlichen Arbeitnehmer der Fall war (Peeters et al. 2007, S. 135).
} 
nur etwa $5 \%$ der Teilzeitbeschäftigten betrifft (Peeters et al. 2007, S. 133f.).

Eine weitere Möglichkeit, die Rentenleistungen von Teilzeitbeschäftigten aufzuwerten, besteht darin, nur die besten Jahre für die Berechnung der Renten zu nutzen. Diese Regelung kommt unter anderem Personen zugute, die sowohl Voll- als auch Teilzeit gearbeitet haben. Viele EU-Länder haben diese Art der Berechnung allerdings zugunsten eines Systems, das die Lebensarbeitszeit zugrunde legt, abgeschafft oder zumindest deutlich abgeschwächt (OECD 2007b, S. 56f.).

Eine Umverteilung zwischen hohen und niedrigen Einkommen in beitragsbasierten Systemen kann auch erreicht werden, indem Leistungsbemessungsgrenzen nicht aber Beitragsbemessungsgrenzen, festgelegt werden. Dies ist beispielsweise in Schweden der Fall, allerdings sind dort die Beiträge, die auf Löhne oberhalb der Leistungsbemessungsgrenze zu entrichten sind, geringer (Ståhlberg et al. 2005, S. 67).

\subsection{ZUSATZALTERSSICHERUNGS- SYSTEME DER ZWEITEN UND DRITTEN SÄULE}

Flexibel Beschäftigte sind häufig direkt (Selbstständige oder geringfügig Beschäftigte) oder, im Falle von zu geringen oder unregelmäßigen Löhnen, indirekt von den Zusatzalterssicherungen der zweiten und dritten Säule ausgeschlossen. Peeters et al. (2007) und O'Connell/Gash (2003) zeigen dies in ihren Analysen zu Betriebsrenten für befristet Beschäftigte in Belgien bzw. Teilzeitbeschäftigte in Irland. Antolin (2008, S. 12f.) bestätigt diese Ergebnisse für Systeme der privaten Vorsorge in acht OECDLändern (darunter Finnland, Deutschland, Irland und das Vereinigte Königreich). Teilzeitbeschäftigte (mit Ausnahme Deutschlands) und Selbstständige sowie Gruppen mit geringem Einkommen sparen weniger häufig für Privatrenten als Vollzeitbeschäftigte, abhängig Beschäftigte und Gruppen mit höherem Einkommen. Eine Möglichkeit, diese Diskriminierung zumindest bei den Betriebsrenten aufzuheben, stellen verpflichtende Betriebsrenten für alle Arbeitsmarktgruppen dar. So können beispielsweise in den Niederlanden schon seit 1994 Teilzeitbeschäftigte nicht aus den zusätzlichen Rentensicherungssystemen ausgeschlossen werden, seit 2002 darf auch die Länge des Vertrags kein Ausschlussgrund sein (European Commission 2006a, S. 222). Auch in Belgien gab es in dieser Hinsicht Verbesserungen. Allerdings können flexibel Beschäftigten dennoch bei der Leistungshöhe Nachteile entstehen, wie Peeters et al. (2007, S. 139) für befristet Beschäftigte zeigen. Insgesamt variiert der Deckungsgrad von Betriebsrenten in EU-27-Ländern deutlich. ${ }^{12}$

Umverteilungsspielräume sind bei Betriebsrenten und privater Vorsorge deutlich geringer, da im Gegensatz zu staatlichen Alterssicherungssystemen Arbeitslosigkeit, Krankheit oder Kindererziehungszeiten seltener berücksichtigt werden (Eurofound 2005, S. 47). Es gibt in dieser Hinsicht allerdings auch positive Beispiele. In Estland, Ungarn, Lettland, Polen, der Slowakei und in umfassendem Maße in Schweden, wird zumindest ein Teil der Erwerbsunterbrechungen auch bei den Betriebsrenten als Beitragszeiten angerechnet (Social Protection Committee 2008, S. 35f.). Bei der deutschen „Riesterrente" profitieren Personen mit Kindern und Geringverdiener stärker von der staatlichen Förderung als andere Gruppen (Riedmüller/Willert 2008, S. 51f.).

Ein weiteres Problem stellt insbesondere bei den Betriebsrenten die Portabilität von Rentenansprüchen dar, die häufig von befristet Beschäftigten, teils aber auch von anderen Erwerbsunterbrechern oder Jobwechslern nicht in Anspruch genommen werden kann. Einige Länder (z. B. Dänemark, die Niederlande und das Vereinigte Königreich) haben die Portabilität von Betriebsrenten in den letzten Jahren verbessert, um die Mobilität der Beschäftigten nicht zu behindern, und auf Europäischer Ebene gibt es Vorschläge für eine Richtlinie (European Commission 2006b, S. 12). In Deutschland existieren trotz Verbesserungen mit der Rentenreform von $2001 \mathrm{mit}$ Mindestbeschäftigungszeiten von fünf Jahren und einem Mindestalter von 30 Jahren noch immer strenge Anspruchskriterien bei den Betriebsrenten. ${ }^{13}$

Wie schwerwiegend sich Sicherungslücken in der zweiten und dritten Säule auf die Absicherung flexibel Beschäftigter auswirken, hängt wesentlich vom Gewicht der staatlichen Säule in der Alterssicherung ab. Der Trend in Europa geht aber hin zu einer Stärkung privater Sicherungselemente.

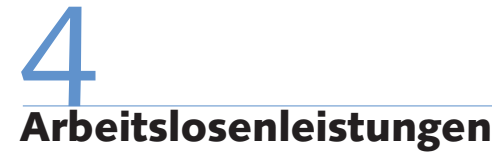

Die folgenden Grundprinzipien von Arbeitslosenversicherungssystemen (ALV) können atypisch Beschäftigte und Personen mit Erwerbslücken beim Zugang zu oder bei der Höhe von Arbeitslosenversicherungsleistungen benachteiligen: Einkommens- oder Stundenschwellenwerte, Anwartschaftszeiten ${ }^{14}$ (Mindestbeitragszeiten innerhalb einer bestimmten Referenzperiode), starke Äquivalenz zwischen vormaligem Lohn und Leistungshöhe ${ }^{15}$ und die Koppelung der Dauer der Leistungsauszahlung an die vorherigen Beitragszeiten.

Eine Studie im Auftrag der Generaldirektion Beschäftigung der Europäischen Kommission kommt basierend auf einer Analyse der Grundprinzipien von ALVSystemen zu dem Schluss, dass ein adäquater und gleichwertiger Zugang zu ALVLeistungen für atypisch Beschäftigte (Teilzeitbeschäftigte, befristet Beschäftigte und Selbstständige) in den EU-15-Ländern stark variiert. So liegt der Deckungsgrad für atypisch Beschäftigte mit unter $30 \%$ in den südeuropäischen Ländern (mit Ausnahme Spaniens) sehr niedrig, während er in den nordischen Ländern (sowie Österreich, Luxemburg und den Niederlanden) $70 \%$ übersteigt (Alphametrics Ltd. 2005, S. 42f.).

Betrachtet man den Zugang von atypisch Beschäftigten zu Arbeitslosenleistun-

12 Länder wie Spanien, Finnland, Luxemburg, Portugal und Polen haben einen Deckungsgrad von unter $10 \%$, während er in den Niederlanden und Schweden bei über 90 \% liegt (Social Protection Committee 2008, S. 10f.).

13 Trotz deutlicher Zuwächse hatte 2003 nur jede fünfte Frau gegenüber mehr als der Hälfte der Männer in Westdeutschland einen Anspruch auf eine Betriebsrente, was auch auf die Geschlechterunterschiede in der Verteilung auf Branchen und Betriebe mit unterschiedlichen Größen zurückführbar ist. Außerdem waren die Betriebsrenten von westdeutschen Frauen deutlich geringer als die von Männern (Klammer 2005, S. 356f.).

14 Bei den Anwartschaftszeiten für ALV-Leistungen werden ähnlich wie bei den Rentenanwartschaften in einigen Ländern Kinderbetreuungszeiten angerechnet.

15 Die Äquivalenz zwischen Lohn und Leistungshöhe ist in Ländern wie Deutschland aufgrund hoher Leistungsobergrenzen vergleichsweise stark, während z. B. in Dänemark und den Niederlanden die Lohnersatzraten mit zunehmendem Einkommen sinken (Leschke 2008, S. 121f.; Schulze Buschoff/Protsch 2008, S. 61, 65). 
gen (Versicherungsleistungen und nachrangige Leistungen) exemplarisch für Dänemark, Deutschland, Spanien und das Vereinigte Königreich - Länder, die sich in der Ausgestaltung und Zielsetzung ihrer Arbeitslosenleistungssysteme deutlich unterscheiden, - zeigt sich, dass Teilzeitbeschäftigte in allen vier Ländern deutlich seltener Zugang zu Arbeitslosenleistungen haben als Vollzeitbeschäftigte. Als besonders problematisch erweisen sich Einkommens- oder Stundenschwellenwerte, die in einer Reihe von EU Ländern existieren (MISSOC 2008a, S. 16). Mit Ausnahme des Vereinigten Königreichs, in dem die Anwartschaft nicht allein durch die Versicherungsdauer, sondern gleichzeitig durch die Einkommenshöhe bestimmt wird, weisen deskriptive Ergebnisse auch auf Benachteiligungen von befristet Beschäftigten beim Zugang zu Arbeitslosenleistungen hin. Allerdings sind die Unterschiede in den Leistungsdeckungsraten von Beschäftigten mit befristeten und unbefristeten Verträgen weniger groß als zwischen Teilzeitund Vollzeitbeschäftigten (Leschke 2008, S. 161f.). Während die absolute Leistungshöhe für Teilzeitbeschäftigte deutlich niedriger ist als für Vollzeitbeschäftigte, sind ihre Nettlolohnersatzraten in allen vier Ländern deutlich höher (Leschke 2008, S. 186f.). Es findet dementsprechend über verschiedene Mechanismen (z. B. Pauschalleistungen in Großbritannien; in Deutschland unter bestimmten Bedingungen Bemessung des Arbeitslosengeldes nach dem früheren Vollzeiterwerbseinkommen ${ }^{16}$ ) eine Umverteilung statt. Die Leistungshöhe zwischen vormals befristeten und unbefristeten Arbeitnehmern unterscheidet sich in Dänemark, Deutschland und im Vereinigten Königreich kaum, wohingegen in Spanien, wo der Anteil befristet Beschäftigter besonders hoch ist, vormals befristet Beschäftigte deutlich niedrigere Leistungen beziehen (Leschke 2008, S. 189f.). Dies kann unter anderem, insbesondere bei häufigen Wechseln zwischen befristeter Beschäftigung und Arbeitslosigkeit, durch das Verfehlen der Mindestversicherungszeiten für ALV-Leistungen erklärt werden. Die Leistungen in der nachgelagerten Arbeitslosenhilfe erfolgen pauschal und auf deutlich niedrigerem Niveau.

Während die Reformen des niederländischen Arbeitslosenversicherungssystems ${ }^{17}$ Ende der 1980er und Anfang der 1990er Jahre explizit zum Ziel hatten, flexibel Beschäftigte (und insbesondere
Teilzeitbeschäftigte) besser zu integrieren (Koopmans et al. 2005, S. 365f.), zielte in Dänemark, Deutschland, Spanien und dem Vereinigten Königreich kaum eine Reform der letzten 15 Jahre explizit auf atypisch Beschäftigte. Im Gegenteil, einige der Reformschritte, die auf die finanzielle Nachhaltigkeit der Systeme ausgerichtet waren, haben die Versicherungssituation atypisch Beschäftigter weiter verschlechtert. Beispiele sind etwa die Abschaffung der Arbeitslosenhilfe in Deutschland, striktere Anwartschaftszeiten für Arbeitslose z. B. in Dänemark, Deutschland und Spanien, Schweden, Ungarn und Island sowie die Verkürzung der Dauer von nicht-bedarfsgeprüften Leistungen in Deutschland und im Vereinigten Königreich.

\section{5 \\ Ausblick}

Flexible Erwerbsverläufe mit Erwerbsunterbrechungen und Phasen atypischer Beschäftigung haben insbesondere aufgrund der steigenden Erwerbstätigkeit von Frauen in den letzten Jahrzehnten an Bedeutung gewonnen. Ihr Ausmaß variiert abhängig vom institutionellen Kontext und den vorherrschenden Normen und Werten stark zwischen den europäischen Ländern.

In Abhängigkeit von den Leitprinzipien der sozialen Sicherungssysteme gibt es bedeutende Länderunterschiede in der Absicherung flexibel Beschäftigter. Während insbesondere die nordischen Länder, aber auch die Niederlande mit ihren individualisierten Versicherungssystemen, die vergleichsweise großzügige erwerbsverlaufsunabhängige Mindestsicherungselemente beinhalten, relativ gut auf flexible Erwerbsverläufe reagieren können, sind die stark auf Äquivalenz setzenden Systeme der kontinentaleuropäischen Länder bislang weniger geeignet, die Risiken flexibel Beschäftigter abzusichern. Vielmehr sind beispielsweise im deutschen System aufgrund des noch immer hohen Anteils abgeleiteter Leistungen nicht oder geringfügig beschäftigte Ehepartner (üblicherweise Frauen) häufig besser abgesichert als flexibel Beschäftigte. Abgeleitete Leistungen sind nicht nur kostenaufwendig, da ihnen keine Beitragseinnahmen gegenüberstehen, sondern sie setzen zusammen mit steuerlichen Vorteilen aufgrund des Ehegattensplittings gleichzeitig Anreize, auf eine reguläre Be- schäftigung zu verzichten, und stärken somit die traditionelle Rollenverteilung in Paarhaushalten.

Wie kann es den kontinentaleuropäischen Sozialversicherungsländern gelingen, vor dem Hintergrund internationaler Erfahrungen bessere Lösungen für die Absicherung flexibler Erwerbsbiografien zu finden? Eine Einschränkung abgeleiteter Leistungen und steuerlicher Vorteile für Ehepaare würde Mittel freisetzen, die zu einer Stärkung umverteilender Elemente in den sozialen Sicherungssystemen genutzt werden und dazu beitragen könnten, flexible Erwerbsverläufe besser abzusichern. Gleichzeitig sollte der bereits eingeschlagene Weg, die Klein- und Schulkinderbetreuung zu verbessern, zügig weiter verfolgt werden, um Eltern eine freie Wahl zwischen Voll- und Teilzeitbeschäftigung und bei der Dauer von Erwerbsunterbrechungen zu erlauben. In diesem Zusammenhang sollten auch die Anreize für Männer, sich stärker an Haushalts- und Betreuungsaufgaben zu beteiligen, weiter gestärkt werden. Ein gutes Beispiel in dieser Hinsicht sind die nichtübertragbaren „Vätermonate" im neuen deutschen Elterngeldrecht, die weiter ausgebaut werden könnten.

Die bisherigen Bestrebungen in Deutschland, die sozialen Sicherungssysteme an flexible Erwerbsverläufe anzupassen, resultierten in einer Reihe von Sonderregelungen wie der Anrechnung von Beitragszeiten bei Erwerbsunterbrechungen, der Aufwertung von Beiträgen aus Phasen der Teilzeitbeschäftigung oder der Aufwertung von Arbeitslosenversicherungsleistungen für bestimmte Gruppen von Teilzeitbeschäftigten. Diese Regelungen sind teilweise intransparent. Und obwohl sie in einigen Bereichen zu Verbesserungen beigetragen haben, reichen sie nicht aus, die großen Unterschiede, etwa zwischen Männern und Frauen, im Zugang zu und in der Höhe von Renten- und Arbeitslosenversicherungsleistungen auszugleichen. Einer der Gründe dafür ist, dass die Aufwertung von Beiträgen häufig nur auf der Basis von Mindestbeiträgen erfolgt und sich, zumindest in

16 Mehr Informationen zu dieser Regelung in Klammer/Leiber (2006, S. 288).

17 Die Anwartschaftszeiten wurden nach den Reformen in Abhängigkeit von Arbeitswochen und nicht wie vorher von Arbeitstagen berechnet. Außerdem wurden 1991 die Stundenschwellenwerte abgeschafft. 
der deutschen Rentenversicherung, auf die erste Säule des Sicherungssystems beschränkt.

Wie könnten also Handlungsempfehlungen für die Bereiche Rentenversicherung und Arbeitslosenversicherung aussehen? In der ersten Säule der Rentenversicherung könnten Grundsicherungselemente weiter gestärkt werden. Diese sollten aber als individualisierte Grundrente und nicht, wie es momentan mit der deutschen Grundsicherung der Fall ist, als bedarfsgeprüfte, stigmatisierende Sozialhilfeleistung gewährt werden. Wichtig ist auch, dass die Grundrente in voller Höhe unabhängig von der Art des vormaligen Beschäftigungsstatus gewährt wird, da sie sonst, wie etwa in Belgien, Ungleichheiten im Arbeitsleben reproduziert. Des Weiteren ist insbesondere die weit verbreitete Stärkung der zweiten und dritten Säule bei gleichzeitiger Stärkung der Äquivalenz zwischen Beiträgen und Leistungen in der ersten Säule problematisch. So beziehen Personen mit kontinuierlichen Erwerbsbiografien und stabilen (hohen) Einkommen nicht nur höhere staatliche Renten, sondern profitieren auch stärker von den Leistungen aus den Zusatzversicherungen. Reformen in diesem Bereich könnten darin bestehen, die Unternehmen zu verpflichten, betriebliche Vorsorge unabhängig vom Erwerbsstatus anzubieten. Kurzfristige Erwerbsunterbrechungen könnten ähnlich wie in der ersten Säule überbrückt werden und die Portabilität von Betriebsrenten sollte deutlich verbessert werden. Auch die dritte Säule könnte stärker verpflichtend gestaltet werden, wobei eine finanzielle Unterstützung von Geringverdienern und Personen mit Kindern wie bei der Riesterrente sinnvoll erscheint.

In der Arbeitslosenversicherung sollten insbesondere Stunden- oder Einkommensschwellenwerte abgesenkt oder abgeschafft werden. Dies würde nicht nur die finanzielle Tragfähigkeit der Systeme erhöhen und breiteren Gruppen den Zugang zu
Leistungen gewähren, sondern gleichzeitig Arbeitgebern und Arbeitnehmern den Anreiz nehmen, geringfügige Beschäftigung anzubieten. Für befristet Beschäftigte erweisen sich insbesondere lange Mindestbeitragszeiten innerhalb kurzer Referenzperioden als problematisch. Arbeitgeber, die statt auf „hire-and-fire“-Politiken auf interne Anpassung über Arbeitszeitkonten oder Trainingsmaßnahmen setzen, sollten finanziell und organisatorisch besser unterstützt werden. Demgegenüber könnte eine Abgabe erwogen werden, die Arbeitgeber, die exzessiven Gebrauch von befristeter Beschäftigung oder Zeitverträgen machen, finanziell an den erhöhten Ausgaben für Arbeitslosigkeit beteiligt. Individuelle Leistungen der Arbeitslosenversicherung sollten ausreichend lange und bevorzugt unabhängig von der vormaligen Beitragszeit gewährt werden und frühzeitig mit aktiven Maßnahmen, insbesondere der Unterstützung bei der Arbeitssuche, verknüpft werden. 


\section{LITERATUR}

Alphametrics Ltd. (2005): Feasibility Study: Indicators on coverage of social security systems for people in flexible employment, Study undertaken by Alphametrics Ltd. for DG-Employment

Antolin, P. (2008): Coverage of Funded Pension Plans, OECD Working Paper on Insurance and Private Pensions 19, Paris

Bruning, G./Plantenga, J. (1999): Parental leave and equal opportunities: experiences in eight European countries, in: Journal of European Social Policy 3, S. 195-209

Bundesagentur für Arbeit (2006): Arbeitsmarktpolitik, Beschäftigungspolitik und Erwerbstätigkeit: Entwicklungen und Zusammenhänge 20002005, Nürnberg

Bundesagentur für Arbeit (2007): Bericht der Statistik der BA: Mini- und Midijobs in Deutschland, Nürnberg

Bundesministerium für Familien, Senioren, Frauen und Jugend (BMFSFJ) (2002): Rentenratgeber für Frauen, Berlin

European Foundation for the Improvement of Living and Working Conditions (2005): Fourth European Working Conditions Survey, Dublin European Commission (2003): Employment in Europe, Brussels European Commission (2006a): Adequate and sustainable pensions. Synthesis report 2006, Luxembourg: Office for Official Publications of the European Communities, http://ec.europa.eu/employment_social/ spsi/docs/social_protection/2006/rapport_pensions_final_en.pdf (Zugriff April 2009)

European Commission (2006b): Commission Staff Working Document. Annexes to the Communication from the Commission to the Council, the European Parliament, the European Economic and Social Committee and the committee of the Regions: Joint Report on Social Protection and Social Inclusion 2006 - Synthesis report on adequate and sustainable pensions, SEC(2006) 304

Europäische Kommission (2004): Angemessene und nachhaltige Renten. Gemeinsamer Bericht der Kommission und des Rates, Amt für amtliche Veröffentlichungen der Europäischen Gemeinschaften, Luxemburg Eurofound (2005): Working time options over the life course: Changing social security structures, Office for Official Publications of the European Communities, Luxembourg.

Eurostat (2008): Labour Force Survey, http://epp.eurostat.ec.europa.eu/ portal/ (Zugriff April 2009)

Klammer, U. (2005): Soziale Sicherung, in: Bothfeld, S./Klammer, U./ Klenner, Ch./Leiber, S./Thiel, A./Ziegler, A. (Hrsg.): WSI-FrauenDatenReport 2005. Handbuch zur wirtschaftlichen und sozialen Situation von Frauen, Berlin, S. 307-382

Klammer, U./Leiber, S. (2006): Atypische Beschäftigung und soziale Sicherung, in: WSI Mitteilungen 5, S. 287-292

Koopmans, I. /Plantenga, J./Vlasblom, J. D. (2005): Life Courses, Diversity and the Reform of the Unemployment Insurance Act, in: European Journal of Social Security 4, S. 363-377

Leschke, J. (2008): Unemployment insurance and non-standard employment: four European countries in comparison, Wiesbaden

Leschke, J./Jepsen, M. (2009): Transitional Labour Markets, from theory to policy application. Can transitional labour markets contribute to a less traditional gender division of labour?, CES Working Papers 2009.27, Paris
Mutual Information System on Social Protection (MISSOC) (2008a): MISSOC Analyse 2008: Sozialschutz: Aspekte von Flexicurity und aktiver Eingliederung, http://ec.europa.eu/employment_social/missoc/2008/ missoc_2008_analysis_de.pdf (Zugriff April 2009)

Mutual Information System on Social Protection (MISSOC) (2008b): Comparative Tables on Social Protection, Situation on 1/7/2008, http://ec.europa.eu/employment_social/missoc/db/public/ compareTables.do? (Zugriff April 2009)

O'Connell, P./Gash, V. (2003): The Effects of Working Time, Segmentation and Labour Market Mobility on Wages and Pensions in Ireland, in: British Journal of Industrial Relations 1, S. 71-95 OECD (2001): Balancing Work and Family Life: Helping Parents into Paid Employment, Employment Outlook, Paris, S. 129-166 OECD (2007a): Babies and bosses: reconciling work and family life. A synthesis of findings for OECD countries, Paris OECD (2007b): Pensions at a Glance: Public policies across OECD countries, Paris

OECD (2009a): Family Database: LMF4: Employment profiles over the life-course (aged 20 to 64), www.oecd.org/els/social/family/database (Stand 11.06.2007)

OECD (2009b): Family Database: PF11: Enrolment in day-care and preschools, www.oecd.org/els/social/family/database (Stand 19.12.2008) O'Reilly, J./Bothfeld, S. (2002): What happens after working part time? Integration, maintenance or exclusionary transitions in Britain and western Germany, in: Cambridge Journal of Economics 26, S. 409-439 Palmer, E. (2001): The New Swedish Pension System, Discussion Paper 36, Institute of Economic Research, Hitotsubashi University Parsons, D. O./Tranaes, T./Bie Lilleør, H. (2003): Voluntary Public Unemployment Insurance, The Danish National Institute of Social Research, Copenhagen

Peeters, H./Debels, A./Verschraegen, G./Berghman, J. (2007): Flexicurity in Bismarckian Countries? Old Age Protection for Non-standard Workers in Belgium, in: Journal of Social Policy 1, S. 125-143

Riedmüller, B./Willert, M. (2008): Die Zukunft der Alterssicherung. Analyse und Dokumentation der Datengrundlagen aktueller Rentenpolitik, Gutachten im Auftrag der Hans-Böckler-Stiftung, Düsseldorf Schulze Buschoff, K./ Protsch, P. (2008): (A-)typical and (in-)secure? Social protection and "non-standard" forms of employment in Europe, in: International Social Security Review 4, S. 51-73

Social Protection Committee (2006): Current and prospective theoretical pension replacement rates, report by the Indicators Sub-Group (ISG) of the Social Protection Committee (SPC), May 19th 2006, http://ec.europa. eu/employment_social/social_protection/docs/isg_repl_rates_en.pdf (Zugriff April 2009)

Social Protection Committee (2008): Privately managed funded pension provision and their contribution to adequate and sustainable pensions, http://ec.europa.eu/employment_social/spsi/docs/social_protection_ commitee/final_050608_en.pdf (Zugriff April 2009)

Ståhlberg, A./Kruse, A./Sundén, A. (2005): Pension Design and Gender, in: European Journal of Social Security 1, S. 57-79 